\title{
Kinetics, Dynamics and Energy of Solid on the Example of a Tool Fixed Flexibly: Part 1 - Kinetics
}

\author{
Zdzisław Pluta, Tadeusz Hryniewicz* \\ Koszalin University of Technology, Raclawicka 15-17, 75-620 Koszalin, Poland \\ *E-mail: Tadeusz.Hryniewicz@tu.koszalin.pl
}

\begin{abstract}
This work investigates kinetics, dynamics and energy of solid on the example of a tool fixed flexibly under the process of cutting. The original approach to the tool kinetics was considered by the Authors earlier. This work consists with three parts referred to the kinetics, dynamics, and energy of solid. Present work is concerned on the development of kinetics problems of a solid represented by a tool fixed flexibly and is a continuation of the problem. Part 1 covers the definition and characteristics of the machining space-time and is referred generally to the kinetics. Then the kinetic and dynamic magnitudes characterizing tool in the space-time are described. The set of these magnitudes has been extended by introducing the properly understood impulse and time-effect. Part 2 of the work is to consider the dynamics of tool in the machining space-time. In Part 3, types of works in the machining space-time and energy of the tool fixed flexibly will be considered; the focus is to be put on an essential difference between work and energy.
\end{abstract}

Keywords: Kinetics; Tool; Machining space-time; Potential field; Potential; Mechanics

\section{INTRODUCTION}

For the introduction, the Authors' work [1] is referred to, concerning an adequate approach to cutting by the tool fixed flexibly. That paper was focused on the kinetics only so the development of the work seems to be a natural need. This work is aiming at the consecutive parts of physical characteristics, such as kinetics, dynamics and energy of the tool. The next step would be characteristics of the system with a cutter fixed together with an elastic element to present a complexity of the problem. Therefore the description of dynamic behaviour of the tool with the state energetic characteristics is to be also presented.

The presented in [1] kinetics of the tool fixed flexibly covers first two links, that is velocity and acceleration. Now the third kinetic link, that is jerk as the derivative of acceleration with respect to time, is to be considered. That will allow to extend description of the tool dynamics. By regarding the tool mass one may obtain consecutive, a properly understood magnitude, being the time-effect.

Therefore the velocity, acceleration, and jerk - all they will be multiplied by the solid/ tool mass to obtain impetus, force of inertia, and time-effect/spur-of-the-moment. It is worth noting that the considered magnitudes will be determined quantitatively both analytically and 
graphically, whereas their units will be resulting from the units of three basic magnitudes, which are the mass, length, and time. Their units are kilogram $(\mathrm{kg})$, meter $(\mathrm{m})$, and second $(\mathrm{s})$, respectively.

Naturally, in the vertical direction of the machining system, there are actions with the measures of other forces, namely repel (repulse or rebuff), and gravity [2,3]. These forces will be also determined.

In this place it is worth defining the notion of the machining system. One should notify that the two elements are the tool and machined material. They are a part of the whole technological system, namely the machine tool - fixture/chuck - workpiece - tool $[1,4]$.

To clarify the notion of vertical direction of the machining system one should assume that each machining system is characteristic with the following directions: vertical and two horizontal. These directions, in turn, are characteristic with the coordinate system which is surely connected with the machining system to allow a description of the magnitudes characterizing the machining process. The most characteristic direction of the considered system is vertical direction.

A closer explanation of the time-effect notion will be provided in the context of tool dynamics. The same explanation of the inertia notion, referred to the flexibly fixed cutter, will be also given. Stored energy of the tool will be provided on the basis of energy notion, the properly understood mechanical energy of a solid/material body. An important task is to provide descriptions of the phenomena on adequately defined fundamental notions.

Taking advantage of the present knowledge, concerned with the dynamics of solid, one may describe the dynamic behavior of the tool fixed flexibly but this description would not be adequate. It results from using the methodic tool which is a d'Alembert's rule [5]. It is known that in the structure of that rule there is a fictional component, being the fictional inertia force.

Therefore a new method of solving the dynamics of the considered solid is needed, with the solid being cutting tool fixed flexibly. The source now will be not the equation of equilibrium of forces (fictional and real ones) but the equation of the path length of the tool edge.

To clarify more strictly the title word referred to the way of a solid/tool fixation (fixed flexibly) one should state that such a manner of its attachment/fixation is characteristic with a possible deflection of tool (and the tool edge together) against a nominal path of cutting which is the horizontal straight line.

\section{CHARACTERISTICS OF THE (MACHINING) SPACE-TIME}

The work covers dynamics and stored energy of the solid/tool fixed flexibly which moves (with one-side free motion) upward due to a repel action of the machined material. In this direction (vertical direction) the kinetics referred to the tool will be considered.

One should stress again that the tool itself is moving upward with the motion of a free character that is the free motion in vertical direction, the motion not dumped by any material external stimulus. (Naturally, the air resistance, left out of account as small, has been omitted in these considerations).

The nature of repel action of the machined material is such that it repels the tool upward. Of course, the tool behaves reversely, that is it press against the material. The behaviour of both cooperating elements of the machining system is in agreement with the commonly recognized third Newton's law [6]. 
It is worth explaining that the second element of the machining system, which is the workpiece, cannot be characterized by magnitudes connected with dynamics. That workpiece does not move with a variable motion which - including the workpiece mass - gives evidence of dynamics of that element. One may speak of kinematics of the workpiece.

The considered machining system, described in detail in [1], consists of edge fixed articulatedly supported tool, as well as the workpiece having the shape of obliquely truncated prism of rectangular basis, and in the longitudinal cross-section - isosceles trapezoid (Fig. 1). The edge 1 is loaded gravitationally by means of weight 2 , whereas the wedge-shaped workpiece 3 is fixed by the chuck 4 , settled in turn on the machine tool table. The workpiece travels with the velocity $v_{m}$, acting/pressing against the tool edge with its oblique plane. The first butt/contact of the workpiece with the tool edge will follow in a determined point of zero line $0-0$.

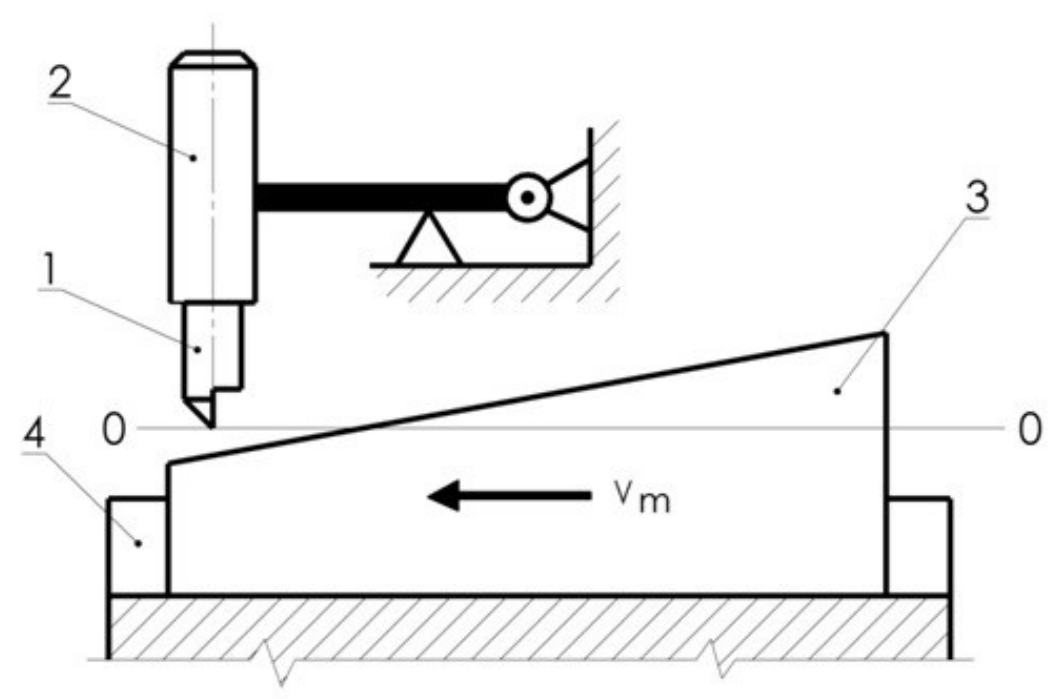

Fig. 1. Structural scheme of a machining system: 1 - edge, 2 - weight, 3 - workpiece, 4 - chuck [1].

Further development of the subject requires explanation of the machining space-time notion, with the space-time itself at the beginning. According to [7] the space-time is a mathematical composition arisen from the junction of one-dimension time and threedimension space into one four-dimension stretch/reach. The work [8] relates that the junction of time and space has been called the space-time. In [9] the space-time is called fourdimension stretch originated as the junction of time and space. The encyclopaedia [10] provides the following definition of the space-time: set of all events; the event is placed in the space-time by providing four coordinates: time and three coordinates determining the position in space.

As it results from the examples of general definitions of space-time, it is characterized by space, time, and one or more events. However, none of those definitions specifies the limits of this composition, though the limits are an important element of determined characteristics of the space-time. They decide of the overall dimensions. The placement of these limits refers to determined energetic states of the solid/material body which penetrates the space-time with a variable motion. The event or phenomenon occurring there, on the 
limits of space-time (here: on the limits situated horizontally) changes its nature, assuming the energetic or state nature. On the horizontal direction, the space-time limits are determined by vertical kinematic potential fields in the extreme points of the second kinetic zone, being the second fragment of the whole machining zone. It will be closer explained in the paper.

Thus the notion of machining space-time has been introduced for the considered technological system. This adjectival determination indicates the material machining takes place in this space-time, meaning a special event occurrence. This event is accompanied by the phenomenon of variable motion, because the solid/tool moves with such a motion during the material machining.

Characteristics of the machining space-time is presented on the background of geometric characteristics of track/trajectory of tool edge within the machined workpiece (Fig. 2). That last characteristics covers two kinetic zones marked by Roman digits I, II. These zones (marked the same) correspond with consecutive fragments of the whole machining zone. In each of them the configuration of edge trajectory is completely different, resulting from its differentiated kinetics. In the first zone (between points 0 and 1), the edge moves on horizontal line, not changing its primary track. On the length of second zone (between points 1 and 2), the edge track declines upward and rises exponentially and progressively which is reflected by the retarded vertical motion of the edge.

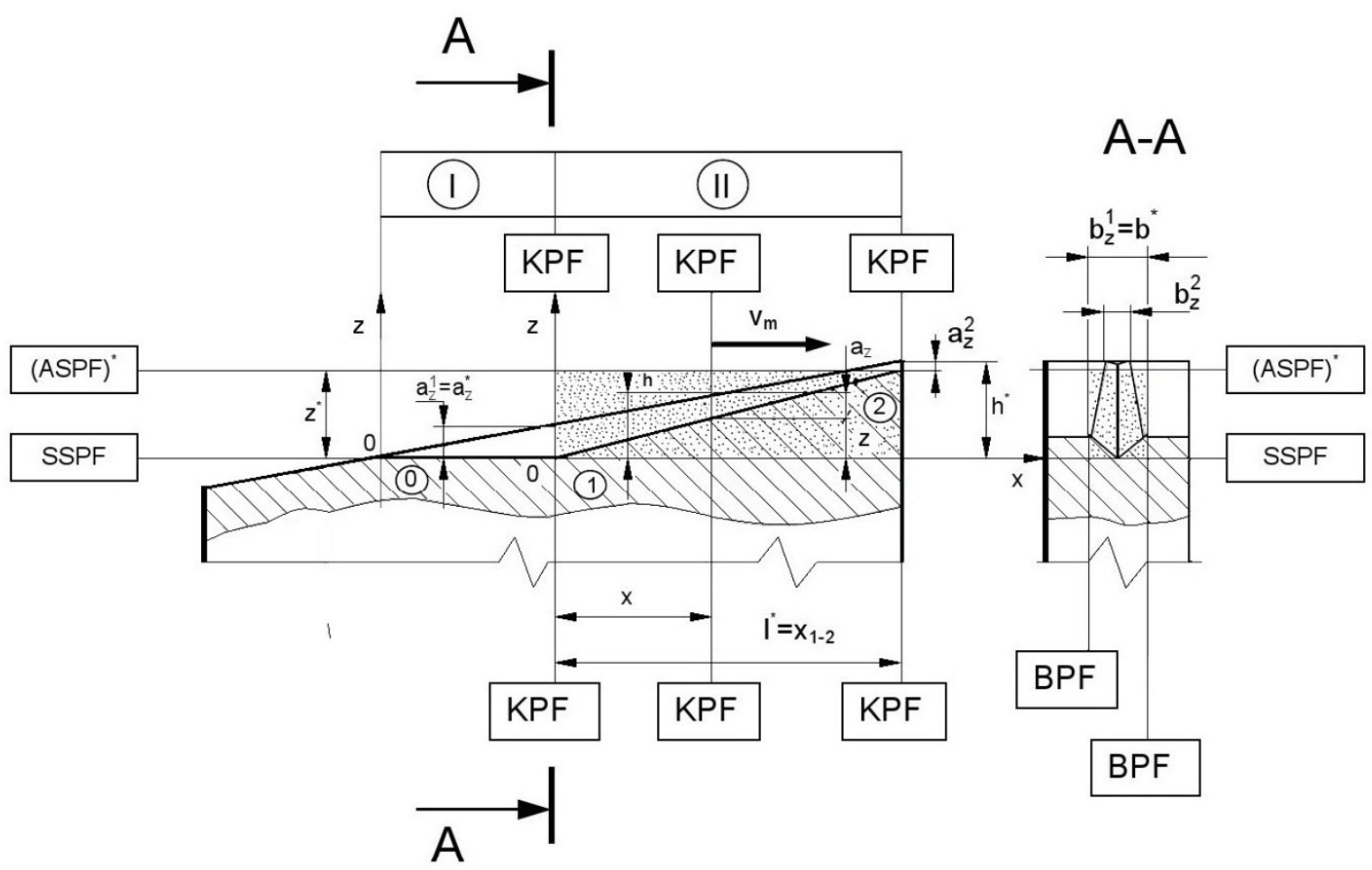

Fig. 2. Geometric characteristics of track/trajectory of edge within the machined workpiece.

These two fragments of the whole machining zone may be called as follows: I - in-cut zone, II - repel zone. The machining space-time (dotted area) is a four-dimension stretch, placed between determined potential fields, where the material treatment takes place by the solid/tool moving with a variable motion. They are three dimensions determining the space where the process takes place, with the fourth dimension being time of the tool motion in the space. 
The space dimensions are determined by the potential fields on which the corresponding energetic states of the tool and workpiece occur. In vertical direction, on the level of zero line (see Fig. 1), there is a stable static potential field SSPF where the edge possesses a stable energetic state. Of course, this field has a time character because the mentioned state lasts longer than a while. At the level/height of place where the edge is leaving material, the instantaneous unstable static potential field $(A S P F)^{*}$ is situated. Here this energetic state in vertical direction lasts for a while so that the field has instantaneous character. The $z^{*}$ distance between these fields, corresponding with the height of edge track, is the height of the space-time. At the same time it is equal to the difference of in-feed $h^{*}$ and the edge in-depth in the place of leaving the material, that is $a_{z}^{2}$. Therefore

$$
z^{*}=h^{*}-a_{z}^{2}
$$

The kinematic potential field, that is $K P F$, is moving in horizontal direction; it is moving in this direction with a constant velocity, being the velocity of machined workpiece material, that is $v_{m}$. Positions of these fields in terminal points of the repel zone are determined by the lengths $l^{*}=x_{1-2}$ of this zone (in horizontal direction).

The third dimension of the space-time (in transversal direction) is illustrated by A-A cross-section of the machined workpiece. In this cross-section, the positions of side/lateral potential fields $S P F$, coming through the terminal points of machining trace in the widest place, are visible. The distance between these fields is the width $b^{*}$ of the space-time, corresponding with the width $b_{z}^{1}$ of the machining trace in the point 1 where the repel zone begins.

It is worth explaining the remaining parameters of the machining trace and a solid/tool edge track. That is the maximum depth $a_{z}^{*}$ of the trace, which is its depth in the point 1 (the place of beginning of the retarded motion of edge in the vertical direction), thus $a_{z}^{1}$. Instantaneous depth $a_{z}$ in the repel zone, that is at the height of coordinate $x$, connected with time $t$ and the velocity $v_{m}$ by the dependence

$$
x=v_{m} \cdot t
$$

is the difference between the instantaneous in-feed $h$ in this zone and the corresponding height of the tool edge track. Therefore

$$
a_{z}=h-z
$$

Symbol $b_{z}^{2}$ determines the width of trace in the point 2 , that is in the site of edge leaving the machined material.

\section{KINETICS OF SOLID/TOOL IN THE MACHINING SPACE-TIME}

Now, having a precise description of the machining space-time notion, as well as its characterization, one may start the description of the tool kinetics in this space-time. The starting point for the kinetics description, and then also dynamics and stored energy of the tool, is the analytically presented dependence of the tool edge path length on time. That 
dependence (together with its mathematical derivation) has been derived and presented in the work [1]. That functional dependence has the following analytical form:

$$
z=2 z^{*}\left(1-e^{-\frac{t}{T_{o}}}\right)
$$

where the symbol $T_{o}$ determines the time constant, corresponding with that retarded motion. Other symbols were explained earlier.

The transition time of the tool edge from the point 1 to point $2, t_{1-2}$, is determined by introducing the parameter $z^{*}$ (second coordinate of point 2) and the mentioned time coordinate to the equation (1.4). Therefore

$$
t_{1-2}=T_{o} \ln 2
$$

and

$$
T_{o}=\frac{t_{1-2}}{\ln 2}
$$

Thus the equation (1.4) is determined mathematically for $t \in\langle 0,+\infty)$, and physically for $t \in\left\langle 0, t_{1-2}\right\rangle$. Of course, only the second interval of time variability will be considered because the adequate and real description of the studied reality is of importance.

The first derivative kinetic magnitude is the tool velocity, that is $v_{z}$. It is the first derivative of the edge path length (also of the whole tool) against time. Thus it is expressed by the following dependence:

$$
v_{z}=\frac{d z}{d t}=\frac{2 z^{*}}{T_{o}} e^{-\frac{t}{T_{o}}}=v_{1}^{0} e^{-\frac{t}{T_{o}}}
$$

Symbol $v_{1}^{0}$ determines the initial vertical velocity, in the point 1 .

In the formula (1.7), taking into account time $t$ resulting from the dependence (1.2), one obtains

$$
v_{z}=\frac{2 z^{*}}{T_{o}} e^{-\frac{x}{v_{m} T_{o}}}=v_{1}^{0} e^{-\frac{x}{v_{m} T_{o}}}
$$

so the velocity as the function of the tool position against the machined workpiece. The product $v_{m} T_{o}$ is the path constant $X_{o}$, referred to the workpiece, that is

$$
X_{o}=v_{m} T_{o}
$$

so

$$
v_{z}=\frac{2 z^{*}}{T_{o}} e^{-\frac{x}{X_{o}}}=v_{1}^{0} e^{-\frac{x}{X_{o}}}
$$


The acceleration is the second derivative kinetic magnitude. It is also the second derivative of the edge path length on time. At the same time, the acceleration is the first derivative of velocity on time. Therefore

$$
p_{z}=\frac{d^{2} z}{d t^{2}}=\frac{d v_{z}}{d t}=\frac{2 z^{*}}{\left(T_{o}\right)^{2}} e^{-\frac{t}{T_{o}}}=\frac{v_{1}^{0}}{T_{o}} e^{-\frac{t}{T_{o}}}=p_{1}^{0} e^{-\frac{t}{T_{o}}}
$$

Symbol $p_{1}^{0}$ denotes the initial vertical acceleration.

Following the way as above, or regarding time expressed by dependence (2) in the formula (1.11), one obtains

$$
p_{z}=\frac{2 z^{*}}{\left(T_{o}\right)^{2}} e^{-\frac{x}{v_{m} T_{o}}}=\frac{v_{1}^{0}}{T_{o}} e^{-\frac{x}{v_{m} T_{o}}}=p_{1}^{0} e^{-\frac{x}{v_{m} T_{o}}}
$$

and further, by regarding (1.9)

$$
p_{z}=\frac{2 z^{*}}{\left(T_{o}\right)^{2}} e^{-\frac{x}{X_{o}}}=\frac{v_{1}^{0}}{T_{o}} e^{-\frac{x}{X_{o}}}=p_{1}^{0} e^{-\frac{x}{X_{o}}}
$$

In turn, the impulse is the third derivative kinetic magnitude. This is the third derivative of the path length against time, the second derivative of velocity against time, and the first derivative of acceleration against time. Taking these into account, one obtains

$$
i_{z}=\frac{d^{3} z}{d t^{3}}=\frac{d^{2} v_{z}}{d t^{2}}=\frac{d p_{z}}{d t}=\frac{2 z^{*}}{\left(T_{o}\right)^{3}} e^{-\frac{t}{T_{o}}}=\frac{v_{1}^{0}}{\left(T_{o}\right)^{2}} e^{-\frac{t}{T_{o}}}=\frac{p_{1}^{0}}{T_{o}} e^{-\frac{t}{T_{o}}}=i_{1}^{0} e^{-\frac{t}{T_{o}}}
$$

where the symbol $i_{1}^{0}$ denotes the initial vertical jerk.

That last magnitude, or jerk, may be also expressed as the function of variable $x$. Therefore

$$
i_{z}=\frac{2 z^{*}}{\left(T_{o}\right)^{3}} e^{-\frac{x}{v_{m} T_{o}}}=\frac{v_{z}}{\left(T_{o}\right)^{2}} e^{-\frac{x}{v_{m} T_{o}}}=\frac{p_{1}^{0}}{T_{o}} e^{-\frac{x}{v_{m} T_{o}}}=i_{1}^{0} e^{-\frac{x}{v_{m} T_{o}}}
$$

By taking into consideration the dependence (1.9), one obtains

$$
i_{z}=\frac{2 z^{*}}{\left(T_{o}\right)^{3}} e^{-\frac{x}{X_{o}}}=\frac{v_{1}^{0}}{\left(T_{o}\right)^{2}} e^{-\frac{x}{X_{o}}}=\frac{p_{1}^{0}}{T_{o}} e^{-\frac{x}{X_{o}}}=i_{1}^{0} e^{-\frac{x}{X_{o}}}
$$

All these kinetic magnitudes have been characterized quantitatively. The values of determined magnitudes were excerpted from [1]. As it was mentioned in the introduction, units of the magnitudes result from the units of the three basic magnitudes, such as mass $(\mathrm{kg})$, length (m), and time (s). The graphic illustration of these magnitudes will be presented as the function of coordinate $x$.

The length of repel zone, or the length of the machining space-time, $x_{1-2}$, equals $90 \cdot 10^{-3} \mathrm{~m}$. The velocity of table on which the sample was fixed, was equal $112 \mathrm{~mm} \cdot \mathrm{min}^{-1}$, or 
$1.8(6) \mathrm{mm} \cdot \mathrm{s}^{-1}=1.86 \cdot 10^{-3} \mathrm{~m} \cdot \mathrm{s}^{-1}$. The in-feed $h^{*}$ equals $7.875 \cdot 10^{-3} \mathrm{~m}$, and the in-depth of edge in the point 2 (at the end of machining zone), or $a_{z}^{2}$, has the value equaling $0.040 \cdot 10^{-3} \mathrm{~m}$. These data will allow to formulate quantitative equation of the edge path length in the repel zone, as well as the equations of remaining kinetic magnitudes: velocity $v_{z}$, acceleration $p_{z}$, and jerk $i_{z}$.

Real height of the repel zone $z^{*}$, corresponding with the height of machining spacetime - according to the formula (1.1) - is equal the difference of in-feed $h^{*}$ and the edge indepth in the site of leaving the material, that is $a_{z}^{2}$. Therefore

$$
z^{*}=h^{*}-a_{z}^{2}=7.875 \cdot 10^{-3}-0.040 \cdot 10^{-3}=7.835 \cdot 10^{-3} \mathrm{~m}
$$

The transition time of edge from the point 1 to point 2 , i.e. $t_{1-2}$, results from the formula (1.2), and is equal

$$
t_{1-2}=\frac{x_{1-2}}{v_{m}}=\frac{90 \cdot 10^{-3}}{1.8(6) \cdot 10^{-3}}=48.387 \mathrm{~s}
$$

Now, the time constant $T_{o}$ should be calculated, and acc. to formula (1.6), it equals

$$
T_{o}=\frac{t_{1-2}}{\ln 2}=\frac{48.387}{0.693}=69.823 \mathrm{~s}
$$

Thus the equation of the path length (1.4) has the following quantitative form:

$$
z==2 z^{*}\left(1-e^{-\frac{t}{T_{o}}}\right)=2 \cdot 7.835 \cdot 10^{-3}\left(1-e^{-\frac{t}{69.823}}\right)=15.67 \cdot 10^{-3}\left(1-e^{-\frac{t}{69.823}}\right)
$$

By substituting the values of the magnitudes $z^{*}, v_{m}$, and $T_{o}$ to the formula (1.8), one obtains

$$
v_{z}=\frac{2 z^{*}}{T_{o}} e^{-\frac{t}{T_{o}}}=v_{1}^{0} e^{-\frac{t}{T_{o}}}=\frac{2 \cdot 7.835 \cdot 10^{-3}}{69.823} e^{-\frac{t}{69.823}}=0.224 \cdot 10^{-3} e^{-\frac{t}{69.823}}
$$

with the unit of $v_{z}$ being $\mathrm{m} \cdot \mathrm{s}^{-1}$.

To determine the quantitative dependence of $v_{z}=f(x)$ the value of path constant $X_{o}$ is needed. It is calculated from the equation (1.9), then

$$
X_{o}=v_{m} \cdot T_{o}=1.8(6) \cdot 10^{-3} \cdot 69.823=130.57 \cdot 10^{-3} \mathrm{~m}
$$

According to equation (1.10), one obtains

$$
v_{z}=\frac{2 z^{*}}{T_{o}} e^{-\frac{x}{X_{o}}}=v_{1}^{0} e^{-\frac{x}{X_{o}}}=\frac{2 \cdot 7.835 \cdot 10^{-3}}{69.823} e^{-\frac{x}{130.57 \cdot 10^{-3}}}=0.224 \cdot 10^{-3} e^{-\frac{x}{130.57 \cdot 10^{-3}}}
$$


That kinetic function has been illustrated graphically in Fig. 3. According to notation/marking of the axes of coordinate system, that function has the following form:

$$
v_{z}=2.24 \cdot 10^{-4} e^{-\frac{t}{69.823}}
$$

or

$$
v_{z}=2.24 \cdot 10^{-4} e^{-\frac{x}{130.57 \cdot 10^{-3}}}
$$

Two these functions have been also placed in Fig. 3.

$$
v_{z}=2.24 \cdot 10^{-4} e^{-\frac{t}{69.823}}
$$

$$
v_{z}=2.24 \cdot 10^{-4} e^{-\frac{x}{130.57 \cdot 10^{-3}}}
$$

$$
\frac{v_{z}}{10^{-4} \mathrm{~m} \cdot \mathrm{s}^{-1}}
$$

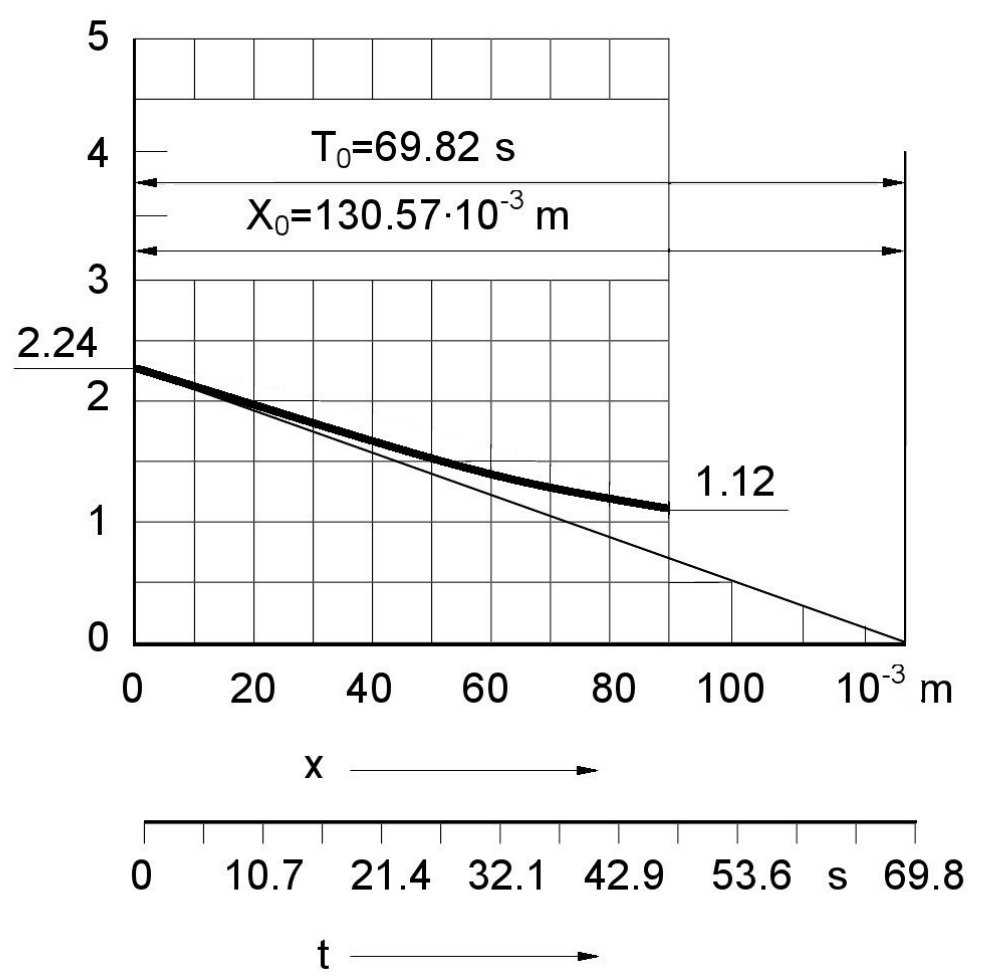

Fig. 3. Dependence of tool velocity $v_{z}$ on time $t$ and horizontal coordinate $x$ of its position on the track of material machining.

The velocity $v_{z}$ of the tool is presented as the function of time $t$ and coordinate $x$ of its position on the path of material treatment. On the direction of horizontal axis the following values are marked: time constant $T_{o}$ and path constant $X_{o}$. As can be seen, they form 
horizontal leg of rectangular triangle, with the vertical leg being the value of the initial velocity $\left(v_{1}^{0}=2.24 \cdot 10^{-4} \mathrm{~m} \cdot \mathrm{s}^{-1}\right)$, and hypotenuse - tangent to the curve $v_{z}=f(x)$ in the initial point.

Now, by substituting values of the magnitudes $z^{*}, v_{m}$, and $T_{o}$ to the formula (1.11), one obtains

$$
\begin{gathered}
p_{z}=\frac{2 z^{*}}{\left(T_{o}\right)^{2}} e^{-\frac{t}{T_{o}}}=\frac{v_{1}^{0}}{T_{o}} e^{-\frac{t}{T_{o}}}=p_{1}^{0} e^{-\frac{t}{T_{o}}}=\frac{2 \cdot 7.835 \cdot 10^{-3}}{(69.823)^{2}} e^{-\frac{t}{69.823}}= \\
=\frac{0.224 \cdot 10^{-3}}{69.823} e^{-\frac{t}{69.823}}=3.208 \cdot 10^{-6} e^{-\frac{t}{69.823}}
\end{gathered}
$$

with the unit of $p_{z}$ being $\mathrm{m} \cdot \mathrm{s}^{-2}$.

After regarding the value $X_{o}$ in the formula (1.13), one obtains

$$
\begin{gathered}
p_{z}=\frac{2 z^{*}}{\left(T_{o}\right)^{2}} e^{-\frac{x}{X_{o}}}=\frac{v_{1}^{0}}{T_{o}} e^{-\frac{x}{X_{o}}}=p_{1}^{0} e^{-\frac{x}{X_{o}}}=\frac{2 \cdot 7.835 \cdot 10^{-3}}{(69.823)^{2}} e^{-\frac{x}{130.57 \cdot 10^{-3}}}= \\
=\frac{0.224 \cdot 10^{-3}}{69.823} e^{-\frac{x}{130.57 \cdot 10^{-3}}}=3.208 \cdot 10^{-6} e^{-\frac{x}{130.57 \cdot 10^{-3}}}
\end{gathered}
$$

That function has been also illustrated graphically in Fig. 4, by presenting the dependence of acceleration $p_{z}$ of the tool on time $t$ and the horizontal coordinate $x$ of its position on the path of material treatment. Visible here the rectangular triangle, with the hypotenuse placed on the direction of tangent to the curve in the initial point, has the vertical leg of right-angled triangle equal to the initial value of acceleration $\left(p_{1}^{0}=3.208 \cdot 10^{-6} \mathrm{~m} \cdot \mathrm{s}^{-2}\right)$. Its horizontal leg is equal to the path constant $X_{o}$, and also corresponding with the time constant $T_{o}$.

At this point, the third kinematic magnitude, i.e. jerk $i_{z}$ will be described. Values of the magnitudes $z^{*}, v_{m}$, and $T_{o}$ are now substituted to the formula (1.14). Therefore

$$
\begin{gathered}
i_{z}=\frac{2 z^{*}}{\left(T_{o}\right)^{2}} e^{-\frac{t}{T_{o}}}=\frac{v_{1}^{0}}{\left(T_{o}\right)^{2}} e^{-\frac{t}{T_{o}}}=\frac{p_{1}^{0}}{T_{o}} e^{-\frac{t}{T_{o}}}=i_{1}^{0} e^{-\frac{t}{T_{o}}}= \\
=\frac{2 \cdot 7.235 \cdot 10^{-3}}{(69.823)^{3}} e^{-\frac{t}{69.823}}=\frac{0.224 \cdot 10^{-3}}{(69.823)^{2}} e^{-\frac{t}{69.823}}= \\
=\frac{3.208 \cdot 10^{-6}}{69.823} e^{-\frac{t}{69.823}}=4.594 \cdot 10^{-8} e^{-\frac{t}{69.823}}
\end{gathered}
$$

with the unit of $i_{z}$ being $\mathrm{m} \cdot \mathrm{s}^{-3}$.

By regarding the values $X_{o}$ in the formula (1.16), one obtains 


$$
\begin{aligned}
& i_{z}=\frac{2 z^{*}}{\left(T_{o}\right)^{3}} e^{-\frac{x}{X_{o}}}=\frac{v_{1}^{0}}{\left(T_{o}\right)^{2}} e^{-\frac{x}{X_{o}}}=\frac{p_{1}^{0}}{T_{o}} e^{-\frac{x}{X_{o}}}=i_{1}^{0} e^{-\frac{x}{X_{o}}}= \\
& =\frac{2 \cdot 7.235 \cdot 10^{-3}}{(69.823)^{3}} e^{-\frac{x}{X_{o}}}=\frac{0.224 \cdot 10^{-3}}{(69.823)^{2}} e^{-\frac{x}{130.57 \cdot 10^{-3}}}= \\
& \frac{3.208 \cdot 10^{-6}}{69.823} e^{-\frac{x}{130.57 \cdot 10^{-3}}}=4.594 \cdot 10^{-8} e^{-\frac{x}{130.57 \cdot 10^{-3}}}
\end{aligned}
$$

$$
p_{z}=3.208 \cdot 10^{-6} e^{-\frac{t}{69.823}}
$$

$$
p_{z}=3.208 \cdot 10^{-6} e^{-\frac{x}{130.57 \cdot 10^{-3}}}
$$

$\frac{p_{z}}{10^{-6} \mathrm{~m} \cdot \mathrm{s}^{-2}}$

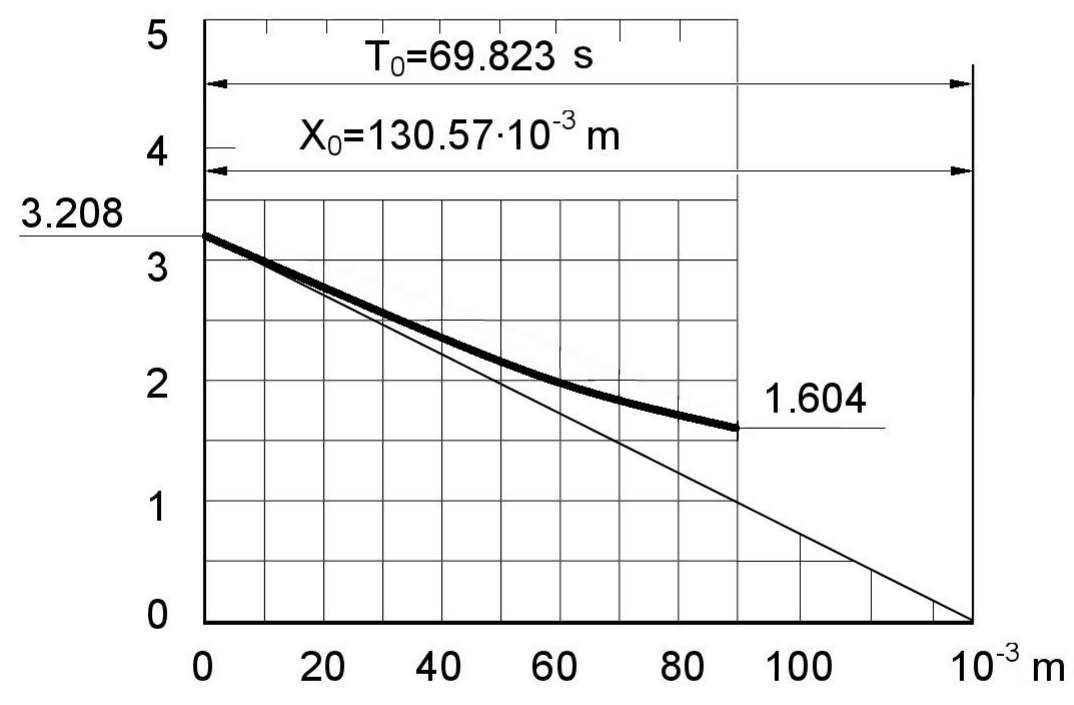

$\mathrm{x} \longrightarrow$

$\begin{array}{llllllll}0 & 10.7 & 21.4 & 32.1 & 42.9 & 53.6 & \text { s } & 69.8\end{array}$

$\mathrm{t}$

Fig. 4. Dependence of acceleration $p_{z}$ of tool on time $t$ and horizontal coordinate $x$ of its position on the path of material treatment

That function has been illustrated graphically in Fig. 5, by presenting the dependence of jerk $i_{z}$ on time $t$ and horizontal coordinate $x$ of its position on the path of material treatment.

The illustration also explains that the curve - alike two former curves - comes out as a tangent from the top of right-angled triangle of the determined values of legs. Here the 
vertical leg is the initial jerk $i_{1}^{0}$ of the value $4.594 \cdot 10^{-8} \mathrm{~m} \cdot \mathrm{s}^{-3}$. The horizontal leg is the path length $X_{o}=130.57 \cdot 10^{-3} \mathrm{~m}$, with the corresponding time constant $T_{o}=69.823 \mathrm{~s}$.

$$
i_{z}=4.594 \cdot 10^{-8} e^{-\frac{t}{69.823}}
$$

$\mathrm{i}_{\mathrm{z}}=4.594 \cdot 10^{-8} \mathrm{e}^{-\frac{\mathrm{x}}{130.57 \cdot 10^{-3}}}$

$$
\frac{i_{z}}{10^{-8} m \cdot s^{-3}}
$$

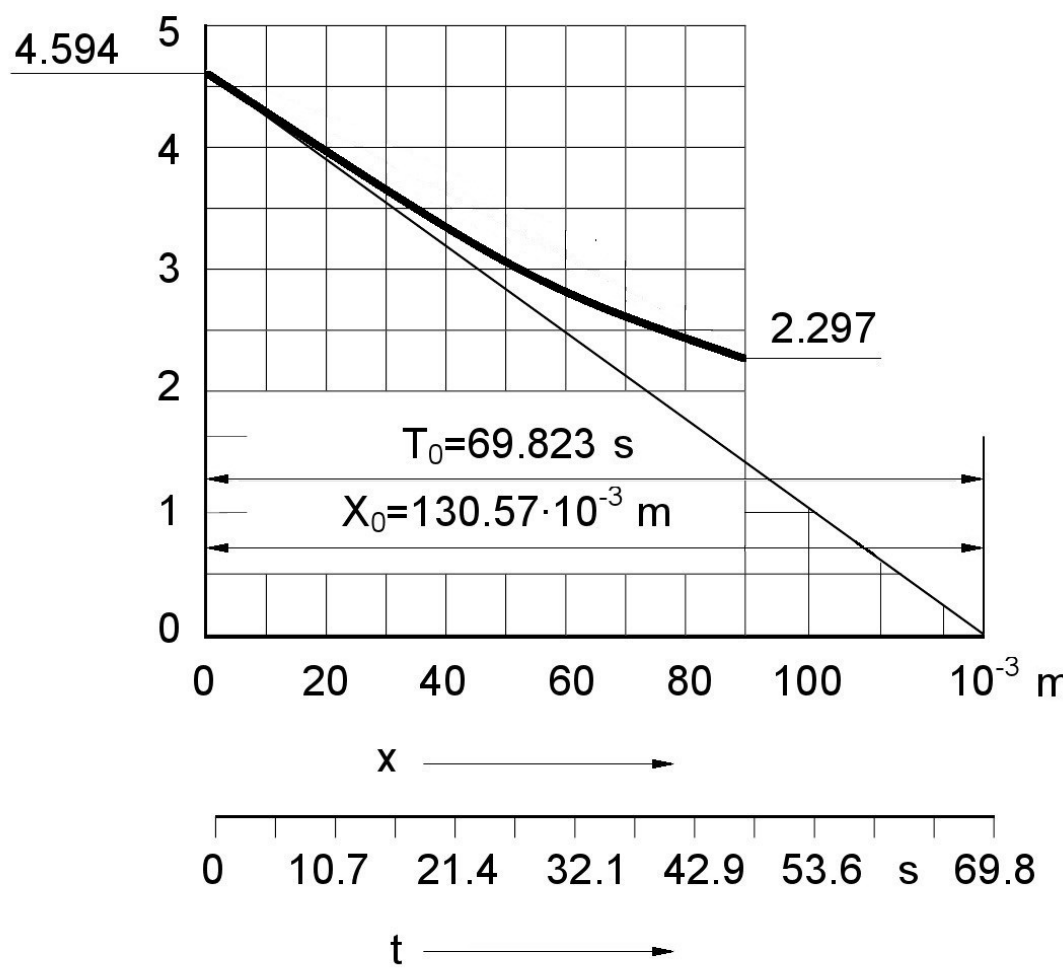

Fig. 5. Dependence of jerk $i_{z}$ of tool on time $t$ and horizontal coordinate $x$ of its position on the path of material treatment.

\section{CONCLUSION}

In the conclusion it is worth underlying the new, quite different method of procedure in reference to the description of the considered system. That method came into being as a result of very critical look on scheme, i.e. the existent rules in the range of description of mechanics of solid. As it results from this work, the behaviour of tool in the machining zone could be described adequately and extensively. It was possible by returning to the source, that is after formulating a correct description of the primary magnitude, being the path length of tool. In turn, that description resulted from the differential equation of a general form, presenting 
dependence of exponential increase/increment of physical magnitudes; the increase/increment resulted from the incremental variations/changes of other magnitudes of this type, is being independent variable. That differential equation was given in [11], and the works [12-15] are to explain other multilateral use of that equation.

This Part 1 concludes the kinetics of the derived system. In Part 2 the dynamics is to be considered, and Part 3 is devoted to the energy of the solid/tool fixed flexibly.

\section{References}

[1] Zdzisław Pluta, Tadeusz Hryniewicz, International Journal of Advanced Manufacturing Technology 62(5) (2012) 529-542; DOI: 10.1007/s00170-011-3813-5.

[2] Zdzisław Pluta, Tadeusz Hryniewicz, International Letters of Chemistry, Physics and Astronomy 4 (2012) 8-16.

[3] Zdzisław Pluta, Tadeusz Hryniewicz, International Letters of Chemistry, Physics and Astronomy 5 (2012) 35-45.

[4] Zdzisław Pluta, Tadeusz Hryniewicz, International Letters of Chemistry, Physics and Astronomy 3 (2013) 67-84.

[5] Zdzisław Pluta, Tadeusz Hryniewicz, International Letters of Chemistry, Physics and Astronomy 7(2) (2013) 85-101.

[6] Simon Saunders, Philosophy of Science 80(1) (2013) 22-48.

[7] M. Heller, Towards the Universe, Editorial House ZNAK, Kraków, 1970, 1st edition.

[8] P. Atkins, Galileo's finger, Editorial House REBIS. Poznań, 2006, 1st edition.

[9] M. Szymczak, Polish vocabulary, Vol. 1, A - K, Warszawa, 1978.

[10] B. Petrozolin-Skowrońska (ed.), Popular Encyclopaedia PWN, Vol. 2, Świat Książki, Warszawa, 1987.

[11] Zdzisław Pluta, Fundamentals of surface smoothing by elastic grinding wheels (in Polish). Edited by Politechnika Koszalińska, Koszalin, 2007.

[12] Zdzisław Pluta, Tadeusz Hryniewicz, International Journal of Advanced Manufacturing Technology 51 (2010) 35-43; DOI: 10.1007/s00170-010-2595-5.

[13] Zdzisław Pluta, Tadeusz Hryniewicz, Journal of Quantum Information Science (JQIS) 1(3) (2011) 127-134; DOI: 10.4236/jqis.2011.13018.

[14] Zdzisław Pluta, Tadeusz Hryniewicz, Journal of Quantum Information Science (JQIS) 1(3) (2011) 149-160; DOI: 10.4236/jqis.2011.13021.

[15] Zdzisław Pluta, Tadeusz Hryniewicz, Tribology Transactions 55(2) (2012) 230-236; DOI: $10.1080 / 10402004.2011 .647385$. 